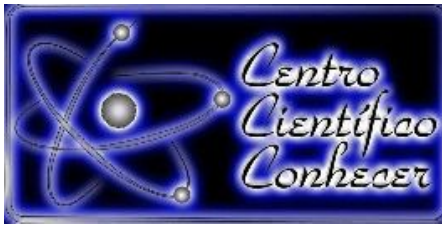

\title{
ESTIMATIVAS DE PARÂMETROS GENÉTICOS EM VARIEDADE DE MANDIOCA SUBMETIDOS À SALINIDADE
}

Leandro Menezes Oliveira ${ }^{1}$, Cláudio Lúcio Fernandes Amaral ${ }^{2}$, Anny Karoline Rocha Quirino $^{3}$, Douglas Gonçalves Guimarães ${ }^{4}$, Murilo Oliveira Guedes ${ }^{5}$

1,3,4 Programa de Pós-graduação em Agronomia, Universidade Estadual do Sudoeste da Bahia, Vitória da Conquista, Bahia, Brasil.

E-mail: leandromenezes_eng@hotmail.com

${ }^{2}$ Departamento de Ciências Biológicas, Universidade Estadual do Sudoeste da Bahia, Jequié, Bahia, Brasil.

${ }^{5}$ Graduando em Agronomia, Universidade Estadual do Sudoeste da Bahia, Vitória da Conquista, Bahia, Brasil.

Recebido em: 20/11/2019 - Aprovado em: 15/12/2019 - Publicado em: 30/12/2019

DOI: 10.18677/Agrarian_Academy_2019b3

A seleção de variedades adaptadas e portadoras de atributos agronômicos desejáveis reveste-se de grande importância para o Nordeste brasileiro onde a maioria dos produtores de mandioca possui capital limitado e não podem investir em tecnologias de produção. Com o objetivo de estimar parâmetros genéticos de características morfológicas e agronômicas de mandioca sob salinidade na água de irrigação, este ensaio foi conduzido em ambiente protegido na Universidade Estadual do Sudoeste da Bahia, campus de Vitória da Conquista. O delineamento utilizado no experimento foi em blocos casualizados, sendo que os tratamentos foram constituídos de cinco variedades (BRS Mulatinha, Platinão, BRS Poti Branca, Sergipe e BRS Verdinha) e quatro níveis de condutividade elétrica $(0 ; 2,0 ; 4,0 ; 6,0$ $\mathrm{dS} \mathrm{m}^{-1}$ ), com quatro repetições, formando um arranjo fatorial $5 \times 4$. Após 120 dias da brotação das manivas foram avaliadas sete características, utilizando estimativas de parâmetros genéticos. Constatou-se alta herdabilidade para todos os traços avaliados, e a maioria apresentou valor alto para ganho genético, favorecendo o melhoramento genético. Em todos os traços avaliados, o coeficiente de variação genotípica foi superior ao coeficiente de variação ambiental, favorecendo a seleção das variedades superiores.

PALAVRAS-CHAVE: Estresse salino, melhoramento genético, recursos genéticos, seleção. 


\title{
ESTIMATES OF GENETIC PARAMETERS IN VARIETIES OF CASSAVA SUBMITTED TO SALINITY
}

\begin{abstract}
The selection of adapted varieties and carriers of desirable agronomic attributes is of great importance to northeastern Brazil, where most cassava growers have limited capital and cannot invest in production technologies. In order to estimate genetic parameters of the morphological and agronomic characteristics of cassava under salinity in irrigation water, this study was conducted in a protected environment at the State University of Southwest Bahia, Vitória da Conquista campus. The experimental design was randomized blocks with five varieties (BRS Mulatinha, Platinão, BRS Poti Branca, Sergipe and BRS Verdinha) and four levels of electrical conductivity $(0 ; 2.0$; 4.0; $6.0 \mathrm{dS} \mathrm{m}-1$ ). ), with 4 replications, forming a $5 \times 4$ factorial arrangement. After 120 days of germination, seven traits were evaluated using estimates of genetic parameters. High heritability was found for all traits evaluated and most presented high value for genetic gain, favoring genetic improvement. In all evaluated characteristics, the genotypic coefficient of variation was higher than the coefficient of environmental variation, favoring the selection of superior varieties.
\end{abstract}

KEYWORDS: Saline stress, genetic resources, breeding, selection.

\section{INTRODUÇÃO}

A mandioca é cultivada principalmente por pequenos agricultores em regiões tropicais e subtropicais, devido as suas raízes serem uma importante fonte de carboidratos (HOWELER et al., 2013). Dentre as culturas comumente produzidas em lavouras no país, é a quarta cultura com maior importância em regiões tropicais (SILVA et al., 2018). Esta se destaca pela característica rústica e grande capacidade de adaptação a condições desfavoráveis de clima e solo, além da vasta abrangência de usos, seja para consumo humano, animal ou industrial (CONAB, 2017).

Em todo território brasileiro é produzida mandioca, com diversos sistemas de produção e diferentes variedades, entretanto a maior parte das variedades utilizadas são nativas bem adaptadas ao ambiente onde estão inseridas, contudo possuem baixo potencial produtivo o que resulta em baixa produtividade nacional (VALLE ; LORENZI, 2014). No nordeste destacam-se como os maiores produtores os estados da Bahia, o Maranhão e o Ceará, que juntos representam cerca de $70 \%$ da produção nordestina. Sendo que o nordeste abrange $37 \%$ da produção nacional. A produção dessa tuberosa em 2017 foi de 20.901 .444 toneladas, redução de $11,8 \%$ frente a 2016 (ANUÁRIO ESTATISTICO DO BRASIL, 2017).

É cultivada em por pequenos agricultores em ambientes estressantes, para o consumo familiar ou para abastecer pequenos negócios locais e regionais (VIEIRA et al, 2013). Oliveira et al. (2018), avaliando o efeito da salinidade nas plantas de

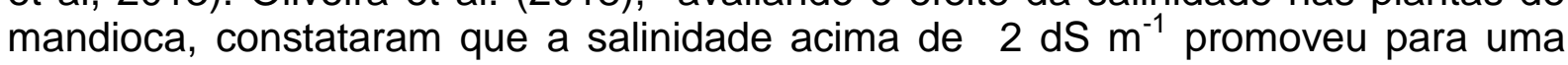
redução considerável na tuberização das raízes, e consequentemente, comprometendo a produtividade.

A seleção de variedades adaptadas e portadoras de atributos agronômicos desejáveis reveste-se de grande importância para o Nordeste brasileiro onde a maioria dos produtores de mandioca possui capital limitado e não podem investir em tecnologias de produção (OLIVEIRA et al., 2018).

Uma ação fundamental no processo de melhoramento genético é a previsão genotípica dos genótipos mais promissores, que depende da estimativa dos 
parâmetros genéticos, bem como nas correlações entre características sob seleção (OLIVEIRA et al., 2015). Recorrendo a análise dos parâmetros genéticos (variâncias fenotípicas, genotípicas e ambientais, coeficientes de variação fenotípica, genotípica e ambiental, herdabilidade, ganho genético), pode-se inferir a variabilidade genética, o grau de expressão de um caráter de uma geração para outra e a possibilidade de ganhos através da seleção direta ou indireta (ROCHA et al., 2003).

Objetivou-se com esse trabalho estimar parâmetros genéticos das variedades de mandioca relacionados a características agronômicas e fisiológicas. O presente estudo visou dar subsídios de escolha de estratégias para o melhoramento genético da cultura.

\section{MATERIAL E MÉTODOS}

O experimento foi conduzido em ambiente protegido, revestido por polietileno e tela lateral, situada na área experimental da Universidade Estadual do Sudoeste da Bahia (UESB), campus de Vitória da Conquista- BA, em uma altitude de aproximadamente 886 metros.

Para o estudo, foram utilizadas cinco variedades, as quais foram obtidas no Banco de germoplasma da UESB, campus de Vitória da Conquista- BA. O delineamento utilizado no experimento foi em blocos ao acaso, sendo que os tratamentos foram constituídos de cinco variedades (BRS Mulatinha, Platinão, BRS Poti Branca, Sergipe e BRS Verdinha) e quatro níveis de salinidade $(0 ; 2,0 ; 4,0 ; 6,0$ $\mathrm{dS} \mathrm{m}^{-1}$ ), com quatro repetições, formando um arranjo fatorial $5 \times 4$.

Neste trabalho, utilizaram-se vasos plásticos com capacidade para 15,6L. Os vasos foram perfurados e envolvidos com sacos plásticos de 50 centímetros de diâmetro, nos quais foram feitos em uma das extremidades um pequeno corte, que foi direcionada para garrafa plástica transparente com capacidade para $2 \mathrm{~L}$, onde foram mensurados os volumes drenados com o intuito de calcular a eficiência do uso da água (EUA), contabilizando somente a água absorvida pela planta.

O substrato para o enchimento dos vasos foi material edáfico não salino, cujas características físico-químicas foram obtidas por análise do solo. Antes da instalação do experimento, todos os vasos foram antecipadamente pesados com o volume do solo determinado para aplicar o método das pesagens. No plantio, as manivas foram dispostas horizontalmente nos vasos com o substrato em profundidade de 10 centímetros, com 15 centímetros de comprimento cada maniva. Durante 30 dias após o plantio (DAP), irrigou-se com a água proveniente da Companhia de Água e Esgoto do Estado da Bahia (EMBASA).

Aos 30 DAP, período em que se alcançou a homogeneidade das plantas no experimento, foi iniciada a irrigação com a solução salina. A irrigação foi efetuada conforme as necessidades hídricas das plantas, estimada de acordo com a capacidade de vaso por intermédio da metodologia desenvolvida por Casaroli e Jong van Lier (2008).

A solução salina foi preparada com o sal $\mathrm{NaCl}$ (sal de cozinha), que foi dissolvido em um recipiente de plástico (capacidade de 80 Litros) contendo água, até se alcançar a condutividade elétrica desejada. A condutividade de cada solução foi mensurada com o auxílio do condutivímetro digital portátil.

Avaliaram-se as características de altura de plantas, Índice de Clorofila Falker (ICF), diâmetro do caule, massa seca das folhas e caules, Razão de Peso Foliar (RPF) e da Eficiência do uso da Água (EUA). Os dados das características foram submetidos a análise de variância, utilizando o programa estatístico Assistat, versão 
Fator de

Quadrados Médios

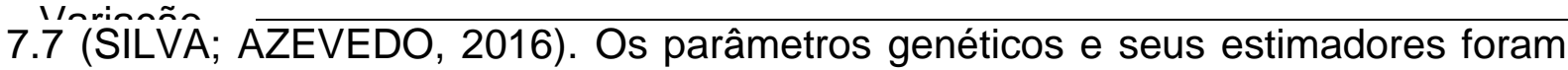
avaliados utilizando-se as seguintes equações (CRUZ et al., 2012).
a) Variância Fenotípica: $V P=\frac{Q M g}{r}$
b) Variância Genotípica: $V G=\frac{Q M g-Q M r}{r}$
c) Variância Ambiental: $V E=\frac{Q M r}{r}$
d) Herdabilidade: $h^{2}=($ VG/VP) $\times 100$
e) Coeficiente de Variação Fenotípica: $C V P=\frac{\sqrt{ } v p}{\mathrm{x}} \times 100$

f) Coeficiente de variação Genotípica: $C V G=\frac{\sqrt{ } V G}{\mathrm{X}} \times 100$

g) Coeficiente de Variação Ambiental: $C V E=\frac{\sqrt{ } V E}{\mathrm{x}} \times 100$

h) Coeficiente de Variação Relativo (Coeficiente "b"): CVR $=\frac{C V G}{C V E}$

i) Ganho genético: $\mathrm{GA}=$ i $\Delta p \mathrm{~h}^{2}$

j) $\mathrm{GAM}=[(\mathrm{GA} / \overline{\mathrm{X}}) \times 100]-$ Ganho Genético em Porcentagem da Média.

\section{RESULTADOS E DISCUSSÃO}

Analisando o resumo da análise de variância do experimento (Tabelas 1), percebe-se que houve diferença significativa entre as variedades avaliadas para todas as sete características avaliadas. Esta situação específica é favorável à seleção de caracteres objetivando o melhoramento genético vegetal. As médias baixas do C.V. foram observadas nas características diâmetro do caule (DC) e altura das plantas (AP), abrangendo valores menores que $20 \%$. Levando em consideração as outras características, todos os valores de C.V.(\%) foram superiores a $20 \%$, sendo: MSC, MSF, RPF, ICF e EUA. Pode-se inferir que as características que apresentaram um valor alto de C.V. (\%), são governadas por muitos genes, por isso as mesmas podem apresentar alta variação.

TABELA 1. Resumo da análise de variância e do coeficiente de variação para altura de plantas (AP), massa seca do caule (MSC), diâmetro do caule (DC), massa seca da folha (MSF), razão do peso foliar (RPF), índice de clorofila falker (ICF), eficiência do uso da água $A$ ) em variedades de mandioca.

\begin{tabular}{ccccccccc}
\cline { 2 - 7 } & MSC & MFC & MSF & AP & RPF & ICF & EUA & DC \\
\hline VP & 3,182 & 63,463 & 2,471 & 0,023 & 0,034 & 72,276 & 0,064 & 0,553 \\
AGRARIAN ACADEMY, Centro Científico Conhecer & - Goiânia, v.6, n.12; & p. 31 & \multicolumn{2}{c}{2019}
\end{tabular}




\begin{tabular}{ccccccccc} 
VG & 2,909 & 60,138 & 1,930 & 0,022 & 0,025 & 47,847 & 0,049 & 0,359 \\
VE & 0,273 & 3,325 & 0,541 & 0,001 & 0,009 & 24,429 & 0,015 & 0,195 \\
VGE & 3,93 & ----- & ---- & ---- & ---- & ---- & ---- & ---- \\
CVP (\%) & 34,703 & 38,747 & 41,041 & 0,447 & 54,233 & 19,165 & 6,253 & 8,917 \\
CVG (\%) & 33,182 & 37,718 & 36,268 & 0,440 & 46,736 & 15,593 & 5,479 & 7,179 \\
CVE (\%) & 10,161 & 8,869 & 19,209 & 0,082 & 27,512 & 11,142 & 3,014 & 5,288 \\
hª (\%) & 91,4 & 94,8 & 78,1 & 96,7 & 74,3 & 66,2 & 76,8 & 64,8 \\
GA & 3,360 & 15,551 & 2,529 & 0,299 & 0,282 & 11,594 & 0,399 & 0,993 \\
GAM & 65,4 & 75,6 & 66,0 & 0,9 & 83,0 & 26,1 & 9,9 & 11,9 \\
CVG/CVE & 3,27 & 4,25 & 1,89 & 5,39 & 1,70 & 1,40 & 1,82 & 1,36 \\
\hline
\end{tabular}

Significativo a $5 \%$ pelo teste $\mathrm{F}(\mathrm{P}<0,05)$

Segundo classificação proposta por Pimentel-Gomes (1990), as características AP e DC apresentam valores médios de C.V. (\%) e as demais características abrangem valores altos de C.V. (\%). Diante disso, pode-se inferir que a seleção será mais favorável quando maior for o C.V.A análise dos parâmetros de variância constatou que a variação fenotípica foi superior a variação genotípica para todas as variáveis estudadas. Resultados análogos foram quantificados por Meira et al. (2017), estudando os parâmetros genéticos na germinação de sementes e emergência de plântulas em feijão-caupi.

TABELA 2. Parâmetros genéticos para altura de plantas (AP), massa seca do caule (MSC), diâmetro do caule (DC), massa seca da folha (MSF), razão do peso foliar (RPF), índice de clorofila falker (ICF), eficiência do uso da água (EUA),em cinco variedades de mandioca.

\begin{tabular}{|c|c|c|c|c|c|c|}
\hline $\begin{array}{l}\text { Fonte de } \\
\text { variação }\end{array}$ & Bloco & Variedade & Salinidade & $\begin{array}{c}\text { Variedade } \mathbf{x} \\
\text { Salinidade }\end{array}$ & Erro & $\begin{array}{l}\text { CV } \\
(\%)\end{array}$ \\
\hline \multicolumn{7}{|c|}{ 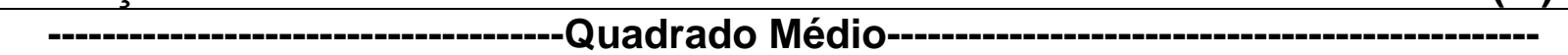 } \\
\hline AP & 0,007823 & $0,090652^{*}$ & $0,130217^{*}$ & 0,005705 & 0,003183 & 16,92 \\
\hline MSC & 1,922327 & $12,727904^{*}$ & $79,279577^{*}$ & $4,206094^{*}$ & 1,091781 & 40,74 \\
\hline DC & 0,612405 & $2,212681^{*}$ & $29,556578^{*}$ & 1,196126 & 0,778335 & 10,57 \\
\hline MSF & 3,657426 & $9,883054^{*}$ & 254,455497 & 2,320374 & 2,165463 & 38,28 \\
\hline RPF & 0,044188 & $0,136772^{*}$ & $0,237^{\star} 897^{*}$ & 0,059829 & 0,035744 & 54,49 \\
\hline ICF & 41,046458 & $289,10393^{* *}$ & 2817,35879 & 185,292854 & 97,717248 & 22,27 \\
\hline EUA & 0,101522 & $0,254239^{*}$ & $9,90^{2 *} 072^{*}$ & 0,058440 & 0,059971 & 30,26 \\
\hline
\end{tabular}

Variação fenotípica (VP), variação genotípica (VG), variação ambiental (VE), variação da interação genótipo $x$ ambiente (VGE), coeficiente de variação fenotípica (CVP), coeficiente de variação genotípica (CVG), coeficiente de variação ambiental (CVE), herdabilidade $\left(h^{2} a\right)$, ganho por seleção (GA), ganho médio por seleção (GAM).

Resultados próximos de CVP E CVE inferem mínimo efeito ambiental, portanto, os valores gerados para estimativas de coeficientes de variação fenotípica e genotípica são atribuídos mais aos fatores genéticos do que ambiental propriamente 
dito. A estimativa de herdabilidade deste estudo foi considerada alta para todos os traços avaliados, com valores variando de 66,2 em ICF até 96,7\% em AP, com 10, das 17 características situadas na faixa entre 70\% a 96\% (Tabela 2). Este indicativo é de suma importância para a seleção destas variedades devido a predominância de ação gênica aditiva (MANGGOEL et al., 2012), sendo indicadores de mínima influência ambiental nas expressões fenotípicas (ARUAH et al., 2012). Valores altos de herdabilidade no sentido geral contribuiram para a transferência das características genéticas dos pais para a prole (RASHWAN, 2010).

Contudo, as estimativas de herdabilidade isoladas não fornecem informações concretas sobre o ganho esperado na próxima geração, mas devem ser associadas em conjunto com as estimativas de avanço genético, a mudança no valor médio entre gerações sucessivas (SHUKLA et al., 2006). Um levantamento da existência da variabilidade genética com o auxílio dos parâmetros adequados, como coeficiente de variação genética, estimativas de herdabilidade e avanço genético é imprescindível para iniciar um programa de melhoramento com eficiência (ATTA et al., 2008).

Para o GAM, observa-se na Tabela 2 que os resultados oscilaram muito em cada característica analisada, a característica que apresentou o menor GAM foi AP, com $0,9 \%$ e a que apresentou o maior valor foi a RPF, com 83,0\%. Segundo a classificação proposta por Sivasubramanian e Menon (1973), cinco características apresentaram o ganho genético alto (MSC, MSF, MFC, RPF, ICF), uma apresentou ganho genético médio (DC) e duas apresentaram baixo ganho genético. Para a relação CVG/CVE, constatou-se que para todos os traços avaliados foi maior do que 1 , com isso, pode-se inferir que o caráter é influenciado mais fortemente pelo fator genético do que pelo ambiental, logo a utilização da seleção torna-se viável.

\section{CONCLUSÕES}

Devido ao fato que foi possível observar altos valores de herdabilidade e ganho genético para características de interesse agronômico, torna-se viável e oportuno selecionar estes materiais para um programa de melhoramento genético.

\section{AGRADECIMENTOS}

Ao programa de pós-graduação por toda a estrutura concedida. À CAPES e a FAPESB pela concessão das bolsas dos pesquisadores.

\section{REFERÊNCIAS}

ANUÁRIO ESTATÍSTICO DO BRASIL. Produção de mandioca 2017. Disponível em:

<ttp://ftp.ibge.gov.br/Producao_Agricola/Fasciculo_Indicadores_IBGE/estProdAgr_20 1701.pdf.> Acesso em:18 de setembro de 2017.

ARUAH, B.C.; UGURU, M.I.; OYIGA, B.C. Genetic variability and inter-relationship among some Nigerian Pumpkin accessions (Curcubita spp.). International Journal of Plant Breeding, v.6, n.1, p.34-41, 2012. Disponível em: https://www.researchgate.net/publication/233529726_Genetic_Variability_and_InterRelationship_among_some_Nigerian_Pumpkin_Accessions_Cucurbita_spp.

ATTA, B.M., HAQ, M.A., \& SHAH, T.M. Variation and inter relationships of quantitative traits in chickpea (Cicer arietinum L.). Pakistan Journal Botany, v. 40, 
n. 2, p. 637-647, 2008. Disponível em: http:// agris. fao.org/ agris-search/ search.do? recordID $=$ PK2010001074.

CASAROLI, D.; JONG VAN LIER, Q. Critérios para determinação da capacidade de vaso. Revista Brasileira de Ciências do Solo, Viçosa, v.32, p.59-66, 2008.Disponível em: http://www.scielo.br/scielo.php?script=sci_arttext\&pid=S010006832008000100007.doi: http://dx.doi.org/10.1590/S0100-06832008000100007.

CONAB - Companhia Nacional de Abastecimento. Conjunturas da agropecuária, Abril 2017. Brasília: CONAB, 2017. Disponível em: http:// www. conab.gov.br/ conteudos.php?a=1253\&t=. Acesso em: 20 setembro de 2017.

CRUZ, C. D.; REGAZZI, A. J.; CARNEIRO, P. C. S. Modelos biométricos aplicados ao melhoramento genético. 4.ed. Viçosa: UFV, 2012, 514 p.

HOWELER, R.; LUTALADIO, N.; THOMAS, G. Save and grow: cassava. A guide to sustainable production intensification. Rome: FAO, 2013. 129p.

MANGGOEL, W.; UGURU, M.I.; NDAM, O.N.; DASBAK, M.A. Genetic variability, correlation and path coefficient analysis of some yield components of ten cowpea (Vigna unguiculata (L.) Walp) accessions. Journal of Plant Breeding and Crop Science, v.4, n.5, p.80-86, 2012. Disponível em: https://www.researchgate.net/ publication/266486047_Genetic_variability_correlation_and_path_coefficient_analysi s_of_some_yield_components_of_ten_cowpea_Vigna_unguiculata_L_Walp_accessi ons.

MEIRA, A. L.; SANTOS, L. G. ; MENDES, H. T. A. E. ; PUBLIO, A. P. P. B. ; SOUZA, U. O. ; Amaral, C. L. F. . Estimativa de parâmetros genéticos para germinação de sementes e emergência de plântulas em feijão-caupi. Scientia agraria paranaensis, v. 16, p. 353-359, 2017. Disponível em: http:// saber. unioeste. br/index. php/ scientiaagraria/article/ download/15074/11826. doi: http://dx.doi.org/10.18188/1983-1471/sap.v16n3p353-359.

OLIVEIRA, E.J. de; AIDAR, S. de T.; MORGANTE, C.V.; CHAVES, A.R. de M.; CRUZ, J.L.; COELHO FILHO, M.A. Genetic parameters for drought-tolerance in cassava. Pesquisa Agropecuária Brasileira, v.50, p.233-241, 2015. Disponível em: http://www.scielo.br/scielo.php?script=sci_arttext\&pid=S0100-

204X2015000300233.doi: 10.1590/ S0100-204X2015000300007.

OLIVEIRA, L. M.; AMARAL, C. L. F.; VIANA, A. E.; CARDOSO, A. D.; GUEDES, M. O.; PESSOA, M. C. B.; PRATES, C. J. N. Variedades de mandioca sob concentrações de salinidade na água de irrigação, em cultivo protegido. Revista de Ciências Agrárias, v. 41, n. 2, p. 251-260, 2018. Disponível em: http://www.scielo.mec.pt/scielo.php?script=sci_arttext\&pid=S0871-

018X2018000200026. Doi: http://dx.doi.org/10.19084/RCA17124.

PIMENTEL-GOMES, F. Curso de estatística experimental. 13.ed. Piracicaba: Nobel, 1990. 468p. 
ROCHA, M. M., CAMPELO, J. E. G., FREIRE FILHO, F. R., RIBEIRO, V. Q., LOPES, A.C. A. Estimativas de parâmetros genéticos em genótipos de feijão-caupi de tegumento branco. Revista Científica Rural, Bagé, v.8, n.1, p.135-141, 2003. Disponível em: http://revistas.ufcg.edu.br/acsa/index.php/ACSA/article/view/817.

RASHWAN, A. M. A. Estimation of some genetics parameters using six populations of two cowpea hybrids. Asian Journal Crop Science, v.2, n.4, p.261-266, 2010. Disponível em: https://scialert.net/abstract/?doi=ajcs.2010.261.267.doi:10.3923/ajcs.2010.261.267.

SHUKLA, S., BHARGAVA, A., CHATTERJEE, A., SRIVASTAVA, A., \& SINGH, S.P. Genotypic variability in vegetable amaranth (Amaranthus tricolor L.) for foliage yield and its ontributing traits over successive cuttings and years. Euphytica, v. 151, p. 103-110, 2006. Disponível em: https: //link. springer. com/ article/10. 1007/ s10681006- 9134-3. doi: 10.1007/ s10681- 006-9134-3.

SILVA, F. A. S.; AZEVEDO C. A. V. The Assistat Software Version 7.7 and its use in the analysis of experimental data. African Journal of Agricultural Research, v. 11, n. 39, p.3733-3740, 2016. Disponível em: https://academicjournals.org/journal/AJAR/article-full-text-pdf/5E8596460818.doi: https://doi.org/10.5897/AJAR2016.11522.

SILVA, L. E. B.; DOS SANTOS, J. K. B.; BARBOSA, J. P. F.; LIMA, L. L. C.; DE SALES SILVA, J. C. Aspectos gerais e peculiaridades sobre mandioca. Diversitas Journal, v.3, n.1, p.1323, 2018. Disponível em: https://periodicos.ifal.edu.br/ diversitas_journal/article/ view/ 587. doi: https://doi.org/10. 17648/ diversitas-journalv3i1.587.

SIVASUBRAMANIAN, S.; P. M. MENON. 1973. Genotypic and phenotypic variability in rice.MadrasAgricultural Journal. J., J, v. 60, n. 9-13, p. 1093-1096, 1973. Disponível em: https://www.researchgate.net/figure/Genotypic-and-phenotypicvariability-in-rice_fig1_302432245.

VALLE, T. L.; LORENZI, J. O. Variedades melhoradas de mandioca como instrumento de inovação, segurança alimentar, competitividade e sustentabilidade: contribuições do instituto agronômico de campinas. Cadernos de Ciência \& Tecnologia, Brasília, v. 31, n. 1, p. 15-34, 2014. Disponível em: https://ainfo.cnptia.embrapa.br/digital/bitstream/ item/102116/1/Variedadesmelhroadas-de-mandioca.pdf.

VIEIRA, E.A.;FIALHO,J.F.; ANDRADE, R. F. R. de. Mandioca no Cerrado Questões práticas. Embrapa - Brasília, 2013. 